PROCEEDINGS OF THE

AMERICAN MATHEMATICAL SOCIETY

Volume 127, Number 7, Pages 1975-1979

S 0002-9939(99)04756-5

Article electronically published on March 17, 1999

\title{
REGULAR CLOSURE
}

\author{
MOIRA A. MCDERMOTT
}

(Communicated by Wolmer V. Vasconcelos)

\begin{abstract}
Regular closure is an operation performed on submodules of arbitrary modules over a commutative Noetherian ring. The regular closure contains the tight closure when both are defined, but in general, the regular closure is strictly larger. Regular closure is interesting, in part, because it is defined a priori in all characteristics, including mixed characteristic. We show that one can test regular closure in a Noetherian ring $R$ by considering only local maps to regular local rings. In certain cases, it is necessary only to consider maps to certain affine algebras. We also prove the equivalence of two variants of regular closure for a class of rings that includes $R=K[[x, y, z]] /\left(x^{3}+y^{3}+z^{3}\right)$.
\end{abstract}

One of our main motivations for studying regular closure is that, unlike the current state of affairs in tight closure theory, regular closure is defined in all characteristics, including mixed characteristic. Also, when an element is in the tight closure of a certain submodule or ideal it is also in the corresponding regular closure. The regular closure contains the tight closure when both are defined, but in general, the regular closure is strictly larger. So, for example, if we could prove that regular closure "captures colons" (see [HH1, (7.6) and (7.14)] for example), then we would be able to prove theorems about maps of Tor vanishing (see [HH1], [HH4]) in mixed characteristic.

Initially, two different notions of regular closure were discussed in the literature (see [HH2], [HH3], [HH4] and [Hu1] for example). This was due, in part, to the lack of a good theory of test elements at that time. One consequence of the theory of test elements is that one can prove that tight closure is preserved by certain base changes [HH5]. This allows one to show that the tight closure of an ideal is contained in one notion of the regular closure of the ideal. We would like to determine when the two notions of regular closure coincide, if at all.

\section{Preliminaries}

Throughout this paper, $R$ denotes a commutative Noetherian ring. Let $R^{\circ}$ denote the complement of the union of the set of elements of minimal primes of $R$.

$\bar{I}$ denotes the integral closure of $I$.

$\mathbb{F}_{p}$ denotes the field with exactly $p$ elements where $p>0$ is a prime.

We now recall the definition of tight closure in characteristic $p>0$ for ideals. Tight closure is defined more generally for modules and also for rings containing

Received by the editors August 7, 1997 and, in revised form, October 20, 1997.

1991 Mathematics Subject Classification. Primary 13A35, 13 H99.

Key words and phrases. Tight closure, regular closure, characteristic $p$.

The author would like to thank the referee for several helpful comments.

(C)1999 American Mathematical Society 
fields of arbitrary characteristic (see [HH1], [HH6] and Appendix 1 of [Hu1]). The reader is referred to $[\mathrm{HH} 1]$ and $[\mathrm{Hu} 1]$ for more information about tight closure.

(1.1) Definition. Let $R$ be a ring of characteristic $p, I$ an ideal of $R$ and $q=p^{e}$ for some non-negative integer $e$. We say that $x \in I^{*}$, the tight closure of $I$, if there exists $c \in R^{\circ}$ such that $c x^{q} \in I^{[q]}$ for all $q \gg 0$ where $I^{[q]}$ denotes the ideal $\left(i^{q}: i \in I\right) R$. If $I=I^{*}$, we say that $I$ is tightly closed.

Note that $I^{*}$ is an ideal of $R$ containing $I$ and every ideal in a regular ring is tightly closed [HH1, (4.1), (4.4)].

\section{Regular Closure}

In the following definition we define two notions of regular closure. It is not known when these two notions are the same if at all.

(2.1) Definition. Let $R$ be a commutative Noetherian ring and $I$ an ideal of $R$. An element $x$ of $R$ is in the regular closure of $I$, denoted $I^{\text {REG }}$, if for every homomorphism of $R$ into a regular ring $S$ which maps $R^{\circ}$ into $S^{\circ}$, the image of $x$ in $S$ is in the image of $I$ in $S$. If we consider all maps $R \rightarrow S$ with $S$ regular but without the requirement that $R^{\circ}$ maps into $S^{\circ}$, then $x \in I^{\text {reg }}$ if the image of $x$ in $S$ is in the image of $I$ in $S$.

The ideal $I^{\text {reg }}$ is the unique largest ideal not distinguishable from $I$ by mappings of $R$ to regular rings.

(2.2) Comment. Clearly $I^{\text {reg }} \subseteq I^{\mathrm{REG}}$. It is also true that $I^{*} \subseteq I^{\mathrm{REG}} \subseteq \bar{I}$ whenever tight closure is defined. The first inclusion is immediate from the definition of tight closure and the fact that every ideal in a regular ring is tightly closed. The second inclusion follows from the fact that integral closure is tested by mapping to DVR's. It is also true that $I^{*} \subseteq I^{\text {reg }}$. We will show that it is sufficient to test regular closure by considering maps to complete regular local rings. If $h: R \rightarrow S$ is a homomorphism of Noetherian rings of characteristic $p$, then if $S$ is a complete local ring and $x \in R$ is in the tight closure of $I \subseteq R$, then $h(x) \in(I S)^{*}$ [HH5]. Since every ideal in a regular ring is tightly closed, we have $I^{*} \subseteq I^{\text {reg }}$.

(2.3) Example. An example which shows that in characteristic $p$ the tight closure can be strictly smaller than the regular closure is $R=K[[x, y, z]] /\left(x^{3}+y^{3}+z^{3}\right)$. One can show that $z$ is in the regular closure of $(x, y) R$ but not in the tight closure $[\mathrm{HH} 4,(5.6)]$ or $[\mathrm{Hu} 1$, Exercise 5.3].

We now discuss some reductions one can make in studying regular closure of ideals. In particular, when testing whether $u \in I^{\text {reg }}$ or $u \in I^{\mathrm{REG}}$ it suffices to look at local homomorphisms into complete regular local rings.

(2.4) Proposition. Let $R$ be a Noetherian ring, $I$ an ideal of $R$ and $u$ an element of $R$. If for every homomorphism of $R$ into a complete regular local ring $S$, the

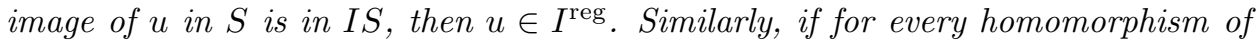
$R$ into a complete regular local ring $S$ which maps $R^{\circ}$ into $S^{\circ}$, the image of $u$ in $S$ is in $I S$, then $u \in I^{\mathrm{REG}}$. If $R$ is a local ring, then it suffices to look at local homomorphisms into complete regular local rings.

Proof. First, it is sufficient to look at maps into regular local rings. Suppose $u \notin$

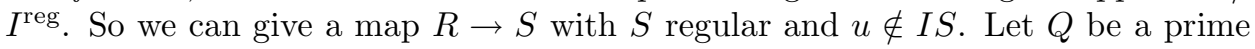


containing $I S: u$. Then $u \notin I S_{Q}$. To see this note that if $u \in I S_{Q}$, then $u$ can be written as $i s / r$ where $i \in I, s \in S$ and $r \in S \backslash Q$. If $u=i s / r$ is in $S_{Q}$, then there exists $d \in S \backslash Q$ with $d(u r-i s)=0$. But this implies that $d u r=d i s$ and hence $d r \in I S: u$. But $d$ and $r$ are both in $S \backslash Q$.

Next we show that it is sufficient to look at homomorphisms into complete regular local rings. Suppose $u \notin I^{\text {reg }}$. So there is a map $R \rightarrow S$ with $u \notin I S$. The map $S \rightarrow \hat{S}$ is flat and local and hence faithfully flat. So $I \hat{S} \cap S=I$. Let $u \in I \hat{S}$; then $u \in I S$ which is a contradiction.

Finally, we show that if $R$ is a local ring, then it is sufficient to consider only local homomorphisms into complete regular local rings. Suppose $S$ is local and $u \notin I^{\text {reg }}$. So there is a map $R \rightarrow S$, not necessarily a local homomorphism, with $u \notin I S$. Again, localize at a prime containing $I S: u$, say $P$. Then $u \notin I S_{P} . I$ maps into $m_{S_{P}}=P S_{P}$ since $I$ maps to $I S \subseteq I S: u \subseteq P$. Let $P^{c}$ denote the contraction of $P$. So $I \subseteq P^{c}$. The map $R_{P^{c}} \rightarrow S_{P}$ is a local homomorphism of local rings. As before, $u / 1 \notin I S_{P}$.

The property that $R^{\circ}$ maps into $S^{\circ}$ is preserved by composition. Also, the property holds if $R \rightarrow S$ is flat. As localization and completion are both flat, we can make the same reductions as above when testing $u \in I^{\mathrm{REG}}$.

(2.5) Discussion. When $R$ is essentially of finite type over a perfect field, we can make further reductions in testing regular closure. Let $R$ be a Noetherian ring essentially of finite type over $K$ where $K$ is a perfect field. By this we mean that $R$ is a localization of a finitely generated $K$-algebra. Let $I \subseteq R$ and $u \in R$ and suppose $u \notin I^{\mathrm{reg}}$. So we have a map $R \rightarrow S$ with $S$ regular and $u \notin I S$. We may reduce to the case where $S$ is a complete regular local ring by Proposition 2.4. As $K$ is perfect, the image of $K$ in $S$ can be enlarged to a coefficient field for $S$, say $K \subseteq L$. Then $S \cong L\left[\left[x_{1}, \ldots, x_{n}\right]\right]$ where the $x_{i}$ are indeterminates.

Suppose $R$ is a localization of $K\left[\theta_{1}, \ldots, \theta_{n}\right]$. Then we have the following maps:

$$
K\left[\theta_{1}, \ldots, \theta_{n}\right] \rightarrow K\left[v_{1}, \ldots, v_{n}\right] \rightarrow L\left[v_{1}, \ldots, v_{n}\right] \subseteq L\left[\left[x_{1}, \ldots, x_{n}\right]\right]
$$

where $v_{i}$ is the image of $\theta_{i}$ in $S$. By a theorem of Artin and Rotthaus [ArR], the map $L\left[v_{1}, \ldots, v_{n}\right] \rightarrow L\left[\left[x_{1}, \ldots, x_{n}\right]\right]$ factors through $L\left[x_{1}, \ldots, x_{n}, y_{1}, \ldots, y_{m}\right]_{(x, y)}^{h}$, where $x, y$ are algebraically independent elements (over $L$ ) of the maximal ideal of $L[[x]]$ and ${ }^{h}$ denotes Henselization. In addition, the map

$$
L\left[x_{1}, \ldots, x_{n}, y_{1}, \ldots, y_{m}\right]_{(x, y)}^{h} \rightarrow L\left[\left[x_{1}, \ldots, x_{n}\right]\right]
$$

is local. If $R$ is actually an affine $K$-algebra, then instead of taking the entire Henselization, we can take a pointed étale extension of $L[x, y]_{(x, y)}$, and, hence, an étale extension of $L[x, y]$, since a pointed étale extension is a direct limit of étale extensions. By another direct limit argument we may also replace $L$ by a regular affine $K$-algebra, so one may test regular closure in this case by mapping only to affine $K$-algebras.

Since the maximal ideal of $L\left[x_{1}, \ldots, x_{n}, y_{1}, \ldots, y_{m}\right]_{(x, y)}^{h}$ contracts to the maximal ideal of $K\left[\theta_{1}, \ldots, \theta_{n}\right]$ and $R$ is a localization of $K\left[\theta_{1}, \ldots, \theta_{n}\right]$, the map $K\left[\theta_{1}, \ldots, \theta_{n}\right] \rightarrow L\left[x_{1}, \ldots, x_{n}, y_{1}, \ldots, y_{m}\right]_{(x, y)}^{h}$ factors through $R$. In other words, we now have a map $R \rightarrow L\left[x_{1}, \ldots, x_{n}, y_{1}, \ldots, y_{m}\right]_{(x, y)}^{h}$ and this is a factorization of the map $R \rightarrow L\left[\left[x_{1}, \ldots, x_{n}\right]\right]$. 
Next we show that we can test regular closure in $K[[x, y, z]] /\left(x^{3}+y^{3}+z^{3}\right)$ using maps to $\mathbb{F}_{p}[A, B, C]_{A B}[[V]] /\left(A^{3}+B^{3}+C^{3}\right)$ where $A, B, C$ and $V$ are indeterminates and the subscript $A B$ denotes localization at the element $A B$.

(2.6) Proposition. Let $R=K[[x, y, z]] /\left(x^{3}+y^{3}+z^{3}\right)$, char $K \neq 3, u \in R$ and $I$ an ideal in $R$. Then $u \in I^{\mathrm{reg}} / I^{\mathrm{REG}}$ if and only if $u \in I T_{A B}$ where $T_{A B}=$ $\mathbb{F}_{p}[A, B, C]_{A B}[[V]] /\left(A^{3}+B^{3}+C^{3}\right)$ and $A, B, C$ and $V$ are indeterminates.

Proof. Let $R^{\prime}=\mathbb{F}_{p}[[x, y, z]] /\left(x^{3}+y^{3}+z^{3}\right)$. First note that the map $R^{\prime} \rightarrow R$ factors through any map $R^{\prime} \rightarrow S$ where $S$ is a regular local ring. There is a natural map $R^{\prime} \rightarrow T_{A B}$ with $x \mapsto A V, y \mapsto B V$ and $z \mapsto C V$. This map factors through any map $R^{\prime} \rightarrow S$ where $S$ is a regular local ring. To see this, we note that any map $R^{\prime} \rightarrow S$ must be of the form $x \mapsto \alpha d, y \mapsto \beta d$, and $z \mapsto \gamma d$ where $d \in S$, $\alpha^{3}+\beta^{3}+\gamma^{3}=0$ and either at least two of $\alpha, \beta, \gamma$ are units or all three elements are $0[\mathrm{HH} 4,(5.6)]$.

To see that $T_{A B}$ is regular, first note that

$$
\begin{aligned}
\mathbb{F}_{p}[A, B, C]_{A} /\left(A^{3}+B^{3}+C^{3}\right) & \cong \mathbb{F}_{p}\left[A, A^{-1}, B / A, C / A\right]_{A} /\left(1+(B / A)^{3}+(C / A)^{3}\right) \\
& \cong \mathbb{F}_{p}[r, s] /\left(1+r^{3}+s^{3}\right),
\end{aligned}
$$

which is certainly regular. So $\mathbb{F}_{p}[A, B, C]_{A B} /\left(A^{3}+B^{3}+C^{3}\right)$ is regular, as is $\mathbb{F}_{p}[A, B, C]_{A B}[[V]] /\left(A^{3}+B^{3}+C^{3}\right)$.

Finally, we show that in certain rings, including $K[[x, y, z]] /\left(x^{3}+y^{3}+z^{3}\right), I^{\text {reg }}=$ $I^{\mathrm{REG}}$ for all $I \subset R$.

(2.7) Proposition. Let $(R, m)$ be a local domain. Suppose the image of $m$ in any power series ring is principal and $B l_{m} R$, the blowup of $m$ in $R$, is regular. Then $I^{\mathrm{reg}}=I^{\mathrm{REG}}$ for all $I \subset R$.

Proof. Let $m=\left(x_{1}, \ldots, x_{n}\right)$. We write $B l_{m} R$ to denote

$$
\bigcup_{j} \operatorname{Spec} R\left[x_{1} / x_{j}, \ldots, x_{n} / x_{j}\right] \subseteq \bigcup_{j} R_{x_{j}}
$$

Suppose $u \notin I^{\mathrm{reg}}$. Then there exists a map $R \rightarrow S$ with $u \notin I S$. As before, we can assume the map is local and $S$ is a complete regular local ring, i.e. $S$ is a power series ring. Since $m S$ is principal, $f\left(x_{i}\right)$ divides $f\left(x_{j}\right)$ for some $i$ and all $j$. Let $f\left(x_{j}\right)=a_{j} f\left(x_{i}\right)$ where the $a_{j}$ lie in $S$.

Next we would like to see that the map $f: R \rightarrow S$ factors through $R\left[x_{j} / x_{i}\right]$. Let $h: R \rightarrow R\left[x_{j} / x_{i}\right]$ be the obvious inclusion. Let $g: R\left[x_{j} / x_{i}\right] \rightarrow S$ be defined as follows: $g(r)=f(r)$ for $r \in R$ and $g\left(x_{j} / x_{i}\right)=a_{j}$. It follows that $f(r)=g(h(r))$ for $r \in R$.

Next we would like to see that $h(u) \notin I R\left[x_{j} / x_{i}\right]$. Suppose $h(u) \in I R\left[x_{j} / x_{i}\right]$. We can write $h(u)=\sum w_{k} i_{k}$ where $w_{k} \in R\left[x_{j} / x_{i}\right]$ and $i_{k} \in I$. Then

$$
g(h(u))=g\left(\sum w_{k} i_{k}\right)=\sum g\left(w_{k}\right) g\left(i_{k}\right)=\sum g\left(w_{k}\right) f\left(i_{k}\right)
$$

since $i_{k} \in R$. So $g(h(u)) \in I S$. But $g(h(u))=f(u)$ and $f(u) \notin I S$. This is a contradiction; hence $h(u) \notin I R\left[x_{j} / x_{i}\right]$. As $h$ is injective and $R\left[x_{j} / x_{i}\right]$ is regular, we have shown that $u \notin I^{\mathrm{REG}}$. So $u \notin I^{\text {reg }}$ implies $u \notin I^{\mathrm{REG}}$ or equivalently, $I^{\mathrm{REG}} \subseteq I^{\mathrm{reg}}$. We already know that $I^{\mathrm{reg}} \subseteq I^{\mathrm{REG}}$, thus $I^{\mathrm{reg}}=I^{\mathrm{REG}}$.

(2.8) Remark. The rings $R=K[[x, y, z]] /\left(x^{3}+y^{3}+z^{3}\right)$, char $K \neq 3$, satisfy the conditions of Proposition 2.4 and so $I^{\mathrm{reg}}=I^{\mathrm{REG}}$ for these rings. 


\section{REFERENCES}

[ArR] M. Artin and C. Rotthaus, A Structure theorem for power series rings, Algebraic Geometry and Commutative Algebra: In honor of Masayoshi Nagata, vol. I, Kinokuniya, Tokyo, 1988, pp. 35-44. MR 90b:14006

[HH1] M. Hochster and C. Huneke, Tight closure, invariant theory, and the Briançon-Skoda theorem, J. Amer. Math. Soc. 3 (1990), 31-116. MR 91g:13010

[HH2] _ Tight closure and elements of small order in integral extension, J. Pure Appl. Algebra 71 (1991), 233-247. MR 92i: 13002

[HH3] _ Tight closure and strong F-regularity, Mémoires de la Société Mathématique de France, numéro 38 (1989), 119-133. MR 91i:13025

[HH4] , Phantom Homology, Memoirs Amer. Math. Soc. Vol. 103, No. 490 (1993), 1-91. MR 93j: 13020

[HH5] _ F-regularity, test elements, and smooth base change, Trans. Amer. Math. Soc. 346 (1994), 1-62. MR 95d:13007

[HH6] _ Tight closure in equal characteristic zero, in preparation.

[Hu1] C. Huneke, Tight closure and its applications, C.B.M.S. Regional Conf. Ser. in Math. No. 88 (1996). MR 96m:13001

Department of Mathematics, Bowdoin College, 8600 College Station, Brunswick, MAINE 04011-8486

E-mail address: mmcdermo@polar.bowdoin.edu

Current address: Department of Mathematics, Gustavus Adolphus College, 800 West College Avenue, St. Peter, Minnesota 56082

E-mail address: mmcdermo@gac.edu 